\title{
Monoenergetic positron conversion in heavy ion fragments
}

\author{
Paul Schlüter and Gerhard Soff \\ Gesellschaft für Schwerionenforschung, Darmstadt, Germany \\ Walter Greiner \\ Institut für Theoretische Physik, Johann Wolfgang Goethe-Universität, Frankfurt am Main, Germany
}

(Received 20 December 1985)

\begin{abstract}
Conversion processes in light nuclei with transition energies above the $\mathrm{e}^{+}, \mathrm{e}^{-}$pair creation threshold are investigated within an analytical framework. In particular, we evaluate the ratio of electron transition probabilities from the negative energy continuum into the atomic $K$ shell and into the positive energy continuum, respectively. The possible role of monoenergetic positron conversion with respect to the striking peak structures observed in $\mathrm{e}^{+}$spectra from very heavy collision systems is examined.
\end{abstract}

At present, the positron emission in elastic and deepinelastic collisions of very heavy ions is the subject of widespread experimental activities. ${ }^{1-7}$ Narrow peak structures at a kinetic positron energy of about $E_{e^{+}}=300 \mathrm{keV}$ were observed $^{1-5}$ at projectile energies close to the nuclear Coulomb barrier. Currently there exists no convincing theoretical explanation of these puzzling cusps which reproduces all experimental facts. Conversion processes in the projectile and target nuclei as a possible source of the detected monochromatic positrons could be ruled out experimentally by direct measurements of the associated photon and $\delta$-electron spectra as well as by a line-shape analysis.

In this note we study the hypothesis that a neck links the projectile and target nucleus at the distance of closest approach. In the course of separation a light ion fragment could be formed with an excitation energy above the pair production threshold. This situation is illustrated in Fig. 1.

The nuclear transition may occur by transferring the energy to an electron in the negative energy continuum, thus yielding a positron. The final state of the electron could be a vacant bound state, preferably the $K$ shell, or a positive energy continuum state. The first of these conversion processes is associated with monoenergetic positron emission. As an example, the ratio of the probabilities of these two different transition modes is calculated assuming a transition energy of $\omega=2.6 m_{e} c^{2}$ in a beryllium nucleus $(Z=4)$. The multipolarities $E 0, E 1, E 2, M 1$, and $M 2$ are considered. The theoretical ingredients concerning internal pair formation are presented in Refs. 8 and 9 and need not be repeated here. We calculated the differential conversion coefficient $d \beta / d E$ for internal $e^{+}, e^{-}$pair creation with respect to the kinetic positron energy $E$, which is defined by

$$
\frac{d \beta}{d E}={\frac{d P_{e}}{d E}}^{+}, e^{-} \frac{1}{P_{\gamma}} .
$$

$P_{e^{+}, e^{-}}$is the probability for electron-positron pair formation. $P_{\gamma}$ denotes the photon emission probability in a nu-

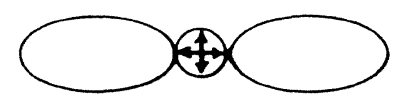

FIG. 1. Schematic representation of the formation of a light ion fragment in the neck connecting two heavy deformed nuclei. clear transition of multipolarity $E L$ or $M L(L \geqslant 1)$ and transition energy $\omega$. In the following, $P_{e^{-}}$signifies the complementary probability for ionizing a $1 s$ electron. The corresponding quantity for nuclear $E 0$ transitions will be denoted by $d \eta / d E$. In this case single photon emission is strictly forbidden.

$$
\frac{d \eta}{d E}=\frac{d P_{e^{+}}}{d E} e^{-} \frac{1}{P_{e^{-}}} .
$$

Neglecting nuclear size corrections and nuclear penetration effects, one may derive ${ }^{10}$ in the limit $Z \alpha \rightarrow 0$ an elementary analytical expression for the positron spectrum resulting from electric monopole transitions $\left(\hbar=c=m_{e}=1\right)$,

$$
\frac{d \eta}{d E}=\frac{p p^{\prime}\left(E E^{\prime}-1\right)}{2 \pi(Z \alpha)^{3} \sqrt{\omega}(\omega+2)^{3 / 2}} .
$$

Here $E$ and $E^{\prime}$ denote the energies of the positron and electron $\left(E+E^{\prime}=\omega\right)$, whereas $p$ and $p^{\prime}$ denote the corresponding momenta. The integration over positron energies $E$ yields

$$
\begin{aligned}
\eta=\int_{1}^{\omega-1} d E \frac{d \eta}{d E} & \\
=\frac{\omega+2}{\omega}\left(\frac{\omega-2}{8 Z \alpha}\right)^{3} & {\left[4{ }_{2} F_{1}\left(-\frac{1}{2}, \frac{1}{2} ; 2 ; x^{2}\right)\right.} \\
& \left.-x_{2} F_{1}\left(-\frac{1}{2}, \frac{3}{2} ; 3 ; x^{2}\right)\right],
\end{aligned}
$$

with the abbreviation

$$
x=\frac{\omega-2}{\omega+2} \text {. }
$$

$\eta l_{s}^{+)}$denotes the ratio of the probability for exciting a negative energy continuum electron to a vacant $K$ shell to the probability for ionizing an electron out of a completely occupied $K$ shell to the positive-energy continuum. We obtain, ${ }^{10}$ in the limit $Z \alpha \rightarrow 0$, the simple result

$$
\eta \hat{s}^{+)}=x^{3 / 2} \text {, }
$$

independent of $Z$. For $\omega=2.6$ and $Z=4$ this leads to

$$
\eta / \eta_{s}^{(+)} \simeq \frac{1}{22(Z \alpha)^{3}} \simeq 2000
$$

In consequence, monoenergetic positron $E 0$ conversion is 


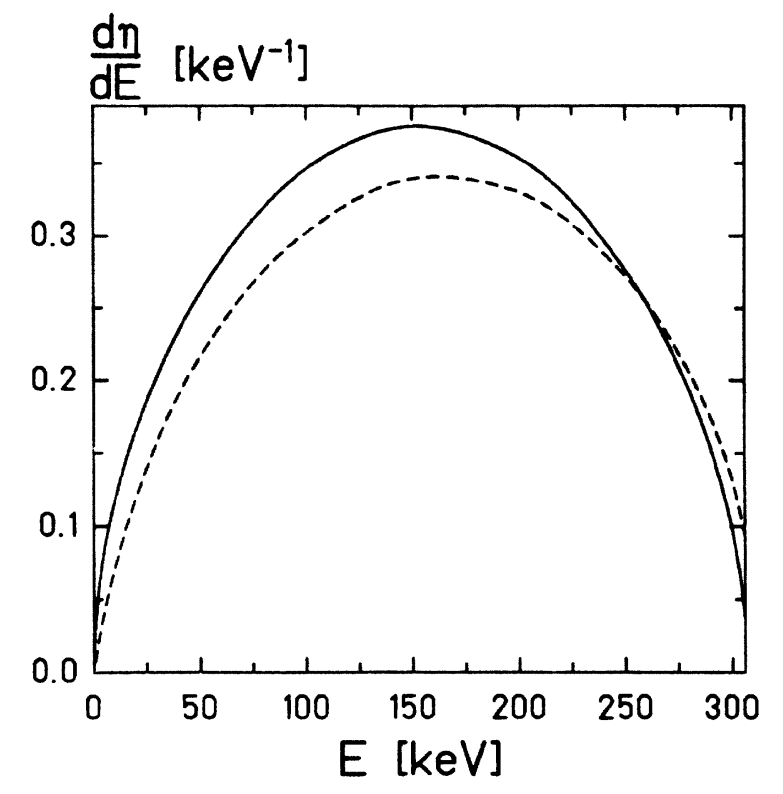

FIG. 2. Differential conversion coefficient $d \eta / d E$ in $1 / \mathrm{keV}$ for an electric monopole transition in beryllium with $\omega=1329 \mathrm{keV}$ vs the kinetic positron energy in keV. Solid line: Analytical result derived in the limit $Z_{\alpha} \rightarrow 0$. Dashed line: Numerical result employing relativistic Coulomb wave functions.

strongly suppressed in light nuclei, which is reflected in the factor $\left(Z_{\alpha}\right)^{-3}$. In completely stripped low- $Z$ ions, nuclear $E 0$ transitions predominantly occur via electron transitions to the positive-energy continuum leading to a continuous $e^{+}$spectrum. In principle, two-photon emission is also allowed. The positron distribution $d \eta / d E$ is displayed in Fig. 2. A broad and flat spectrum (solid line) is obtained, being

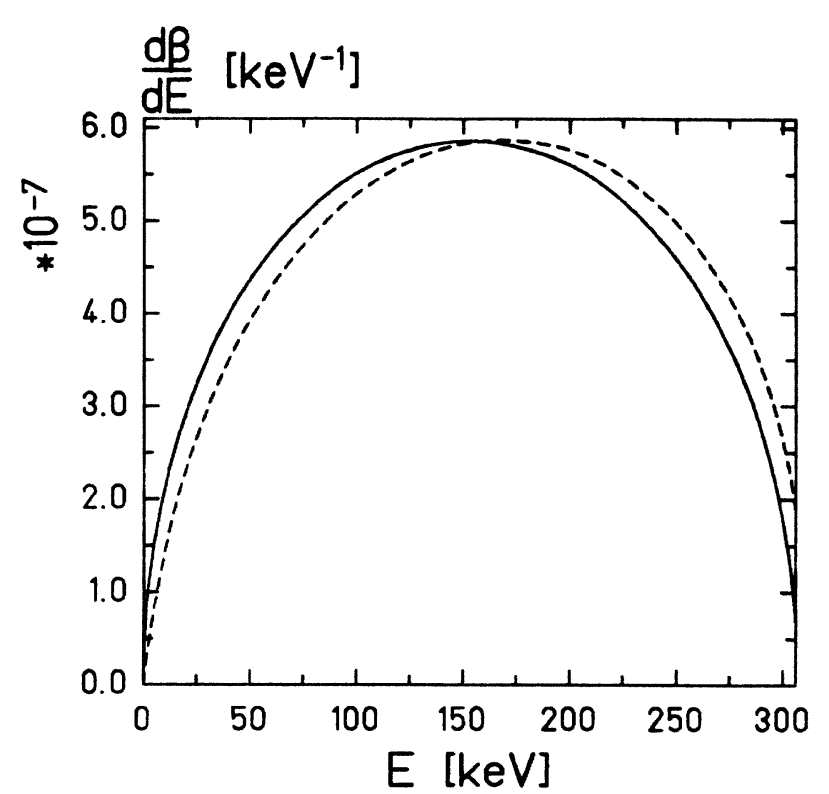

FIG. 3. Differential conversion coefficient $d \beta / d E$ in $1 / \mathrm{keV}$ for electric dipole transitions in beryllium with $\omega=1329 \mathrm{keV}$ vs the kinetic positron energy in $\mathrm{keV}$. Solid line: Analytical result derived within the Born approximation $(Z=0)$. Dashed line: Result employing relativistic Coulomb wave functions.
TABLE I. Total conversion coefficients $\beta$ and $\alpha^{( \pm)}$for $\omega=1329$ $\mathrm{keV}$ in beryllium and various multipolarities. Asymptotic expressions valid for $Z_{\alpha} \rightarrow 0$ are used in the computations. $(x E-n$ denotes $x \times 10^{-n}$ ).

\begin{tabular}{lcccc}
\hline \hline & $E 1$ & $E 2$ & $M 1$ & $M 2$ \\
\hline$\beta$ & $1.42 E-4$ & $3.47 E-5$ & $2.34 E-5$ & $6.39 E-6$ \\
$\alpha^{(+)}$ & $8.69 E-8$ & $2.16 E-8$ & $1.55 E-8$ & $3.58 E-9$ \\
$\alpha^{(-)}$ & $2.41 E-7$ & $4.58 E-7$ & $3.28 E-7$ & $5.81 E-7$ \\
\hline \hline
\end{tabular}

typical for results derived within the Born approximation $(Z=0)$. For the integrated conversion ratios it follows $\eta \simeq 87$ and $\eta_{(s}^{(+)} \simeq 0.047$. For comparison, the dashed line shows the calculated positron spectrum employing relativistic electron wave functions for the Coulomb potential of an extended nucleus. Small Coulomb distortion effects are visible.

Now we turn the discussion to nuclear $E L$ and $M L$ transitions with $L \geqslant 1$. In contrast to electric monopole transitions, here single photon emission evidently represents a competing process. The ratio of the electron transition probability from the negative-energy continuum into a vacant $K$ shell and the photon emission probability $P_{y}$ will be denoted by $\alpha^{(+)}$. Similarly, the quantity $\alpha^{(-)}$determines the ordinary $K$-shell conversion from the completely occupied bound state to the positive-energy continuum. Thus, $\alpha^{(+)}$is correlated with monoenergetic positron production, whereas $\alpha^{(-)}$corresponds to monoenergetic electron emission. Again, in the limit $Z \alpha \rightarrow 0$, we can deduce the handy expressions

$$
\begin{aligned}
\alpha^{( \pm)}(E L)= & \frac{2 \alpha(Z \alpha)^{3}}{L+1}(\omega \mp 2)^{L-1 / 2} / \omega^{L+5 / 2} \\
& \times\left[(L+1) \omega^{2}+4 L\right], \\
\alpha^{( \pm)}(M L)= & 2 \alpha(Z \alpha)^{3}(\omega \mp 2)^{L+1 / 2 / \omega^{L+3 / 2} .}
\end{aligned}
$$

$\alpha^{(-)}(M L)$ as well as $\alpha^{(-)}(E L)$, for the limiting cases $\omega \rightarrow 0$ and $\omega \rightarrow \infty$, agree with the results of Ref. 11 .

Considering continuum-continuum transitions, we evaluated the positron spectrum $d \beta / d E$ resulting from electric dipole transitions. In Fig. 3 we compare the outcome (solid line) of Born approximation calculations $(Z=0)$ with numerical data, employing Coulomb wave functions (dashed line). ${ }^{9}$ Again, both distributions are very similar and exhibit no striking structures. Table I provides the total conversion coefficients $\beta$ and $\alpha^{( \pm)}$for various multipolarities. Here the absolute positron production probabilities are suppressed by about four orders of magnitude compared with the allowed photon emission probability. The monoenergetic $e^{+}$-production mode is additionally suppressed by about three orders of magnitude. Consequently, there should be a strongly enhanced photon peak associated with a possible line structure in the positron distribution.

Based on these intensity arguments, we may conclude that the pronounced $e^{+}$-peak structure detected in collisions of very heavy ions most likely is not correlated with monoenergetic positron conversion in light ion fragments.

We are grateful for enlightening discussions with $\mathrm{H}$. Backe, who brought the idea concerning the investigated conversion mode to our attention. 
${ }^{1}$ E. Berdermann, F. Bosch, M. Clemente, F. Güttner, P. Kienle, W. Koenig, C. Kozhuharov, B. Martin, B. Povh, H. Tsertos, W. Wagner, and Th. Walcher, Gesellschaft für Schwerionenforschung, Darmstadt, Report No. GSI-81-2, ISSN 0174-0814, 1980 , p. 128.

${ }^{2} \mathrm{M}$. Clemente, E. Berdermann, P. Kienle, H. Tsertos, W. Wagner, C. Kozhuharov, F. Bosch, and W. Koenig, Phys. Lett. 137B, 41 (1984).

${ }^{3}$ H. Tsertos, E. Berdermann, F. Bosch, M. Clemente, P. Kienle, W. Koenig, C. Kozhuharov, and W. Wagner, Phys. Lett. 162B, 273 (1985).

${ }^{4}$ J. Schweppe, A. Gruppe, K. Bethge, H. Bokemeyer, T. Cowan, H. Folger, J. S. Greenberg, H. Grein, S. Ito, R. Schule, D. Schwalm, K. E. Stiebing, N. Trautmann, P. Vincent, and M. Waldschmidt, Phys. Rev. Lett. 51, 2261 (1983).
${ }^{5}$ T. Cowan, H. Backe, M. Begemann, K. Bethge, H. Bokemeyer, H. Folger, J. S. Greenberg, H. Grein, A. Gruppe, Y. Kido, M. Klüver, D. Schwalm, J. Schweppe, K. E. Stiebing, N. Trautmann, and P. Vincent, Phys. Rev. Lett. 54, 1761 (1985).

${ }^{6}$ H. Backe, P. Senger, W. Bonin, E. Kankeleit, M. Krämer, R. Krieg, V. Metag, N. Trautmann, and J. B. Wilhelmy, Phys. Rev. Lett. 50, 1838 (1983).

${ }^{7}$ R. Krieg, E. Bozek, U. Gollerthan, E. Kankeleit, G. Klotz, M. Krämer, U. Meyer, H. Oeschler, and P. Senger, Nucl. Instrum. Methods Phys. Res., Sect. B 9, 762 (1985).

${ }^{8}$ P. Schlüter, G. Soff, and W. Greiner, Phys. Rep. 75, 327 (1981).

${ }^{9}$ G. Soff, P. Schlüter, and W. Greiner, Z. Phys. A 303, 189 (1981).

${ }^{10} \mathrm{P}$. Schlüter, Gesellschaft für Schwerionerforschung, Darmstadt, Report No. GSI-85-15, ISSN 0171-4546.

${ }^{11}$ S. M. Dancoff and P. Morrison, Phys. Rev. 55, 122 (1939). 